\title{
Assessment of Switching to Suvorexant versus the Use of Add-on Suvorexant in Combination with Benzodiazepine Receptor Agonists in Insomnia Patients: A Retrospective Study
}

\author{
Masakazu Hatano ${ }^{1,2,3}$, Hiroyuki Kamei ${ }^{3}$, Risa Inagaki ${ }^{2}$, Haruna Matsuzaki ${ }^{2}$, Manako Hanya ${ }^{3}$, Shigeki Yamada ${ }^{2}$, \\ Nakao Iwata ${ }^{1}$ \\ Departments of ${ }^{1}$ Psychiatry and ${ }^{2}$ Clinical Pharmacy, Fujita Health University School of Medicine, Aichi, ${ }^{3}$ Office of Clinical Pharmacy Practice \\ and Health Care Management, Faculty of Pharmacy, Meijo University, Nagoya, Japan
}

\begin{abstract}
Objective: Suvorexant is a novel hypnotic drug that does not interact with the conventional $\gamma$-aminobutyric acid (GABA)-A receptor. We investigated the method by which suvorexant was introduced in insomnia patients who were taking benzodiazepine receptor agonists (BzRA).

Methods: This was a retrospective study. We extracted clinical data for patients who were prescribed suvorexant and were already using BzRA. The patients were assigned to two groups, the switching and add-on groups. We assessed the suvorexant discontinuation rate at one month after the prescription of the drug.

Results: One hundred and nineteen patients were assigned to the switching group, and 109 were assigned to the add-on group. The add-on group exhibited a significantly higher all-cause discontinuation rate than the switching group (odds ratio, 2.7; 95\% confidence interval, 1.5 to 5.0; adjusted $p<0.001$ ). Intolerability was a significantly stronger risk factor for suvorexant discontinuation in the add-on group $(22.0 \%$ vs. $7.6 \%, p<0.002)$, and the most common adverse effect was oversedation.

Conclusion: Our results show that the add-on of suvorexant increases the frequency of oversedation compared with switching in insomnia patients that are taking BzRA. However, this was only a preliminary retrospective study, and further studies will be required to confirm our findings.
\end{abstract}

KEY WORDS: Suvorexant; Orexin receptor antagonists; Benzodiazepines; Insomnia; Adverse effects.

\section{INTRODUCTION}

Insomnia is the most common sleep disorder, and its prevalence is reported to be $21 \%$ in the general adult population of Japan. ${ }^{1)}$ In about $70 \%$ of patients, symptoms persist for at least one year, and being female or elderly are risk factors for the condition. ${ }^{2)}$ Various medications, such as benzodiazepines, non-benzodiazepines, melatonin receptor agonists, sedating antidepressants, and antipsychotic agents are used to treat insomnia. ${ }^{3,4)}$ In partic-

Received: February 17, 2017 / Revised: April 5, 2017

Accepted: April 7, 2017

Address for correspondence: Hiroyuki Kamei, PhD

Office of Clinical Pharmacy Practice and Health Care

Management, Faculty of Pharmacy, Meijo University, 150

Yagotoyama, Tenpaku, Nagoya, Aichi 468-8503, Japan

Tel: +81-528392643, Fax: +81-528348090

E-mail: hkamei@meijo-u.ac.jp

ORCID: https://orcid.org/0000-0003-3778-2352 ular, benzodiazepine receptor agonists (BzRA; benzodiazepines and non-benzodiazepines) are often prescribed by both psychiatrists and general practitioners. ${ }^{5,6)}$ These drugs are easier to use than traditional barbiturates because they appear to be less toxic and are less likely to cause dependence. ${ }^{7)}$ However, BzRA are still associated with numerous problems, such as abuse, physical dependence, an increased risk of falling, and mortality. ${ }^{8-10)}$ In addition, there are growing concerns about polypharmacy and high-dose prescriptions. ${ }^{11}$

Suvorexant has a unique mechanism of action; i.e., it is an orexin receptor antagonist that does not interact with the conventional $\gamma$-aminobutyric acid (GABA)-A receptor, and it was approved for use in Japan in 2014 by the Ministry of Health, Labour and Welfare (the Pharmaceuticals and Medical Devices Agency). Orexins are a type of neuropeptide that are produced by neurons in the lateral

(ㄷ) This is an Open-Access article distributed under the terms of the Creative Commons Attribution Non-Commercial License (http://creativecommons.org/licenses/by-nc/4.0) which permits unrestricted non-commercial use, distribution, and reproduction in any medium, provided the original work is properly cited. 
hypothalamus. They help to regulate the sleep-awake cycle. ${ }^{12)}$ Suvorexant blocks the orexin 1 and 2 receptors, which facilitates sleep induction and maintenance. ${ }^{13,14)} \mathrm{A}$ phase 3 trial demonstrated that suvorexant significantly improved subjective total sleep time (sTST) compared with a placebo, and was generally well tolerated. ${ }^{15)}$ It is also worth noting that no rebound insomnia or withdrawal symptoms occurred when the drug was discontinued after chronic use.

Although several studies of suvorexant have been carried out, there is no evidence about the efficacy or tolerability of combining it with another medications. Most insomnia patients are already being prescribed hypnotic drugs, particularly BzRA. When suvorexant is prescribed to patients that are being treated with BzRA, it can be introduced in one of two ways, switching to suvorexant or combining add-on suvorexant with BzRA. Both approaches can have adverse effects; i.e., switching can lead to rebound insomnia caused by the discontinuation of BzRA, and add-on carries a risk of oversedation due to additive effects. In this study, we carried out a retrospective study of suvorexant treatment in patients that were taking BzRA to assess the most appropriate method for introducing the drug.

\section{METHODS}

\section{Study Design}

We retrospectively analyzed the clinical data in the medical records of Fujita Health University Hospital, Japan. Patients who were prescribed suvorexant between March 2015 and August 2016 while using BzRA were enrolled. The exclusion criteria included an unknown medication history, loss to follow-up, recovery within one month of the start of suvorexant treatment, and enrollment in another clinical trial. The patients were assigned to one of two groups: the switching or add-on group depending on the method used to introduce suvorexant. The switching group was defined as the group in which all or some BzRA were switched to suvorexant. The add-on group was defined as the group in which suvorexant treatment was initiated without decreasing the dose of BzRA or discontinuing BzRA.

We extracted the following clinical data at the baseline: age, sex, the dose of suvorexant, the dose and duration of use of BzRA, any other concurrent sedating medications, and psychiatric diagnoses. In addition, we also investigated the dose of BzRA at one month after suvorexant was prescribed. Regarding the dose of suvorexant, we calculated it not as the prescribed dose, but as the actual dose used; i.e., we considered the possibility of a non-approved dose being used because only two doses (15 mg and $20 \mathrm{mg}$ per day) are approved in Japan. We converted BzRA doses to diazepam equivalents. ${ }^{16)}$ The duration of BzRA use was classified into two categories ( $\leq$ or $>$ one year) because it was difficult to obtain detailed clinical data about this topic. Sedating medication was defined as any of the following: trazodone, mianserin, mirtazapine, quetiapine, levomepromazine, and ramelteon. The psychiatric diagnoses described schizophrenia, bipolar disorder, major depressive disorder and others. The prescribing clinical departments were classified into psychiatry and others. The study was approved by the institutional review board of the Fujita Health University School of Medicine (No. HM16-108).

\section{Outcomes}

The primary endpoint was the discontinuation of suvorexant due to any cause at one month. The secondary endpoint was the reason for the discontinuation of suvorexant in each group. The reason for discontinuation was classified into inefficacy and intolerability, and further investigations into the exact reason why the drug was considered to be intolerable were performed. In addition, the exploratory endpoint was the change in the BzRA dose from the baseline at one month and the BzRA discontinuation rate in the switching group.

\section{Statistical Analysis}

The patients' baseline characteristics were assessed using the Wilcoxon rank sum test for continuous variables. Comparisons of percentages were conducted using Fisher's exact test. The association between the method used to introduce suvorexant and discontinuation was analyzed using a logistic regression model including the following baseline covariates: age, sex, the dose of suvorexant, the dose of BzRA in diazepam equivalents, the duration of BzRA use, the concurrent use of any other sedating medications, and whether the patients have psychiatric illness. We estimated the required sample size according to the standard method for logistic regression analysis; i.e., at least 10 outcomes were needed for each in- 


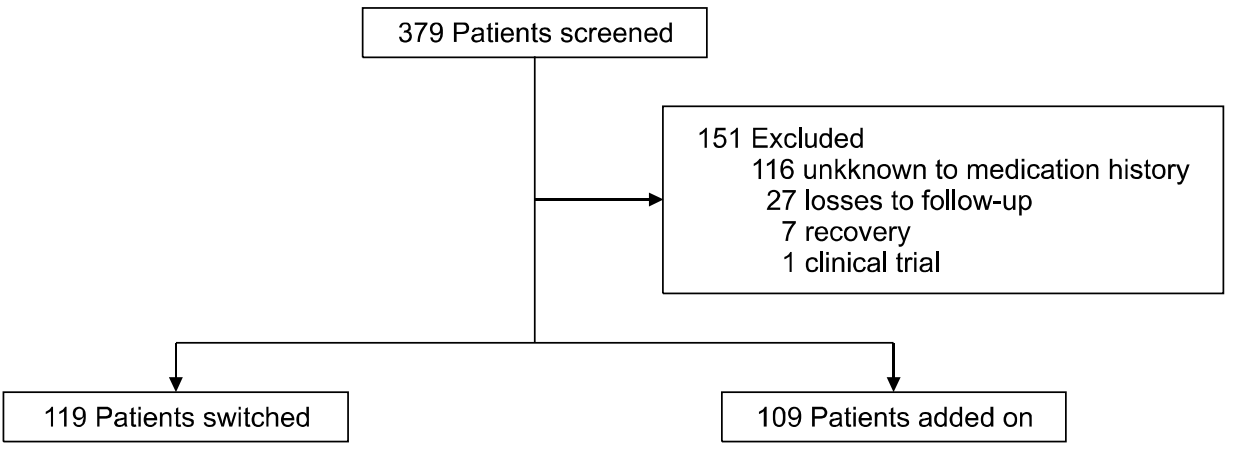

Fig. 1. Flow of patients in this study.

dependent variable. Since we adjusted the seven abovementioned baseline covariates as independent variables in addition to the method used to introduce suvorexant, we required 80 outcomes (discontinuations) to allow us to perform logistic regression analysis. Comparisons of the reasons for the discontinuation of suvorexant were carried out using Fisher's exact test, but only inefficacy and intolerability were subjected to statistical analyses because the sample sizes for the other events were too small. In the switching group, the changes in the doses of BzRA from the baseline were analyzed using the Wilcoxon signed-rank test and Fisher's exact test. The $p$ values of $<0.05$ were considered to be statistically significant. All statistical analyses were performed with EZR (Saitama Medical Centre, Jichi Medical University; http://www.jichi. ac.jp/saitama-sct/SaitamaHP.files/statmedEN.html), which is a graphical user interface for $\mathrm{R}$ (version 2.13.2; The $\mathrm{R}$ Foundation for Statistical Computing, Vienna, Austria). More precisely, it is a modified version of $\mathrm{R}$ commander (version 1.8-4) designed to add statistical functions that are frequently used in biostatistics. ${ }^{17)}$

\section{RESULTS}

\section{Patients' Characteristics}

Of 286 patients screened, 228 met the inclusion criteria (Fig. 1). Of these, 119 patients were assigned to the switching group (males, 46; females, 73; mean age \pm standard deviation $[S D], 52.7 \pm 15.9$ ), and 109 were assigned to the add-on group (males, 44; females, 65; mean age \pm SD, $50.0 \pm 14.7$ ). Six patients used less than the approved dose of suvorexant. The patients' characteristics are summarized in Table 1. None of the examined background characteristics differed significantly between the switching and add-on groups.
Table 1. Baseline patients' characteristic

\begin{tabular}{lccc}
\hline \multicolumn{1}{c}{ Characteristic } & $\begin{array}{c}\text { Switching } \\
(\mathrm{n}=119)\end{array}$ & $\begin{array}{c}\text { Add-on } \\
(\mathrm{n}=109)\end{array}$ & $\begin{array}{c}p \\
\text { value }\end{array}$ \\
\hline Age (yr) & $52.7 \pm 15.9$ & $49.8 \pm 14.7$ & 0.13 \\
Male & $46(38.7)$ & $44(40.4)$ & 0.89 \\
Dose of suvorexant (mg) & $18.4 \pm 2.7$ & $18.7 \pm 2.7$ & 0.32 \\
Diazepam equivalent (mg) & $8.5 \pm 5.8$ & $7.0 \pm 3.7$ & 0.28 \\
Duration of benzodiazepine use & & & \\
$<1$ yr & $34(28.6)$ & $25(22.9)$ & 0.37 \\
$\geq 1$ yr & $85(71.4)$ & $84(77.1)$ & \\
Concurrent sedating medication & & & \\
Trazodone & $11(9.2)$ & $10(9.2)$ & 1 \\
Mianserin & $3(2.5)$ & $6(5.5)$ & 0.32 \\
Mirtazapine & $18(15.1)$ & $18(16.5)$ & 0.86 \\
Quetiapine & $11(9.2)$ & $8(7.3)$ & 0.64 \\
Levomepromazine & $1(0.8)$ & $1(0.9)$ & 1 \\
$\quad$ Ramelteon & $4(3.4)$ & $8(7.3)$ & 0.24 \\
Psychiatric illness & & & \\
Schizophrenia & $12(10.1)$ & $14(12.8)$ & 0.54 \\
Bipolar disorder & $22(18.5)$ & $23(21.1)$ & 0.74 \\
Major depressive disorder & $25(21.0)$ & $34(31.2)$ & 0.10 \\
Others & $22(18.5)$ & $15(13.8)$ & 0.37 \\
\hline
\end{tabular}

Values are presented as mean \pm standard deviation or number (\%). All comparisons between the switching and add-on groups were not significant.

\section{Suvorexant Discontinuation Rate at One Month}

The associations between the suvorexant introduction method and discontinuation are as follows; Regarding the all-cause discontinuation rate, suvorexant was discontinued in $24.4 \%$ (29/119) of the patients in the switching group and $45.0 \%$ (49/109) of patients in the add-on group ( $p<0.001)$. The add-on group exhibited a greater than two and a half times higher risk of suvorexant discontinuation according to the logistic regression model adjusted for seven baseline covariates (adjusted $p<0.001$ ).

The reasons for the discontinuation of suvorexant are summarized in Table 2. Several patients had two or more reasons for discontinuation. The most common reason 
Table 2. Reason for discontinuation of suvorexant

\begin{tabular}{lccc}
\hline \multicolumn{1}{c}{ Event } & $\begin{array}{c}\text { Switching } \\
(\mathrm{n}=119)\end{array}$ & $\begin{array}{c}\text { Add-on } \\
(\mathrm{n}=109)\end{array}$ & $p$ value \\
\hline Inefficacy & $18(15.1)$ & $18(16.5)$ & 0.86 \\
Intolerability & $9(7.6)$ & $24(22.0)$ & 0.002 \\
Somnolence & $5(4.2)$ & $12(11.0)$ & - \\
Fatigue & $1(0.8)$ & $4(3.7)$ & - \\
Nausea & $2(1.7)$ & $2(1.8)$ & - \\
Headache & $0(0.0)$ & $2(1.8)$ & - \\
Abnormal dreams & $1(0.8)$ & $2(1.8)$ & - \\
Allergy & $0(0.0)$ & $3(2.8)$ & - \\
Patient's wishes & $1(0.8)$ & $3(2.8)$ & - \\
Unknown & $3(2.5)$ & $7(6.4)$ & - \\
\hline
\end{tabular}

Values are presented as number (\%).

All events may be duplicated.

Statistical analysis performed only inefficacy and intolerability because the other events is small sample size.

was inefficacy in the switching group (15.1\%) and intolerability in the add-on group (22.0\%). Discontinuation due to intolerability was significantly more common in the add-on group than in the switching $(p<0.002)$. As for the reasons why suvorexant was considered to be intolerable, somnolence was the major reason for discontinuation in both groups.

\section{Change in the Dose of BzRA at One Month}

Table 3 shows the change in the dose of BzRA at one month in the switching group. The BzRA dose in diazepam equivalents was significantly decreased compared with the baseline dose at one month $(p<0.001)$. The mean reduction and the percentage reduction in the BzRA dose in diazepam equivalents were $4.6 \mathrm{mg}$ and $51 \%$, respectively. In total, $46.2 \%$ of the patients in the switching group completely discontinued BzRA.

\section{DISCUSSION}

This is the first study to assess the method used to introduce suvorexant in insomnia patients taking BzRA. The frequency of all-cause suvorexant discontinuation was higher in the add-on group than in the switching group, and discontinuation due to intolerability was significantly more common in the add-on group. A previous metaanalysis of suvorexant monotherapy reported that the all-cause discontinuation rate and the intolerability-related discontinuation rate were $17.9 \%$ and $5.8 \%$, respectively. ${ }^{18)}$ Although it was not possible to perform a stat-
Table 3. Change of BzRA between baseline and at one month in the switching group

\begin{tabular}{lccc}
\hline \multicolumn{1}{c}{ Variable } & Baseline & At a month & $p$ value \\
\hline Diazepam equivalence $(\mathrm{mg})$ & $8.5 \pm 5.8$ & $3.9 \pm 4.9$ & $<0.001$ \\
Discontinuation rate of BzRA $(\%)$ & reference & 46.2 & $<0.001$ \\
\hline
\end{tabular}

Values are presented as mean \pm standard deviation or percent only. BzRA, benzodiazepines receptor agonists.

istical comparison of our findings with those of the latter study because of differences in the study protocols, the rates for both outcomes were clearly high in the add-on group. As for the reasons why suvorexant was considered to be intolerable, the most common adverse effect was somnolence. In addition, fatigue, headaches, and nausea were also experienced by the patients in the add-on group and were probably associated with daytime hangovers. These results suggest that the add-on group suffered oversedation caused by the additive effects of suvorexant and BzRA.

The switching group was considered to be at risk of rebound insomnia and withdrawal symptoms caused by the discontinuation of BzRA. Furthermore, there were concerns about increased discontinuation due to a reduction in efficacy. In the present study, the switching group displayed a higher all-cause discontinuation rate than was reported for suvorexant monotherapy in a previous meta-analysis. ${ }^{18)}$ The risk factors for benzodiazepine dependence are reported to include a high dose and a prolonged treatment duration. ${ }^{19)}$ Although it was not possible to accurately assess the severity of rebound insomnia in this study, it was suspected that many of the patients were in a state of benzodiazepine dependence because they had used BzRA for over a year. Therefore, our results suggest that quick dose reduction and the discontinuation of BzRA when introducing suvorexant may cause rebound insomnia. Conversely, the add-on group exhibited a similarly high inefficacy-related discontinuation rate to the switching group. The add-on group was not considered to be at risk of rebound insomnia from a pharmacological point of view because such patients take stable doses of BzRA. The inclusion criteria for this study restricted the study population to patients who were already taking BzRA; hence, the subjects might have included patients with chronic or refractory insomnia. Furthermore, previous randomized control studies excluded patients without primary insomnia (e.g., those with major affective or 
psychiatric disorders, who were engaging in substance abuse, or who were suffering from unstable medical conditions), whereas our study included all patients that were prescribed suvorexant regardless of their disease. ${ }^{15,20)}$ It is suspected that these study protocol differences are one of the causes of the increased inefficacy-related discontinuation rate seen in this study. Another possibility is that it is difficult to obtain the desired effectiveness when using suvorexant as an add-on to BzRA. However, in the present study we only evaluated effectiveness based on the discontinuation rate, and there is no evidence that combined treatment with suvorexant and BzRA is effective, so the results obtained should be interpreted with caution.

In the switching group, the BzRA dose in diazepam equivalents was significantly lower than the baseline dose at one month after the switch, and almost half of the patients were able to discontinue BzRA. The tapering rate generally fell along with the dose of BzRA. A previous review recommended that reductions of $1 \mathrm{mg}$ every 1-2 weeks are preferred for patients receiving doses of less than $20 \mathrm{mg}$ of diazepam equivalents, as was the case in this study. ${ }^{21)}$ The mean reduction in the dose of BzRA in diazepam equivalents was about $5.0 \mathrm{mg}$ per month, which is indicative of the rapid withdrawal of benzodiazepine. Several drugs have been researched as tools for ameliorating benzodiazepine withdrawal symptoms. ${ }^{22)}$ When pretreatment with trazodone and sodium valproate was employed, about $70 \%$ of patients achieved benzodiazepine withdrawal within five weeks. ${ }^{23)}$ On the other hand, prolonged-release melatonin had no significant effect on the withdrawal of benzodiazepines compared with a placebo ${ }^{24)}$; thus, the use of adjuvant drugs to aid the withdrawal of benzodiazepines requires further study. Suvorexant might be a useful adjuvant drug for aiding the discontinuation of benzodiazepines, but further investigations are required to confirm this.

Our study had several limitations. The patients' clinical data were incomplete, e.g., there was insufficient information about the severity of the patients' insomnia, the efficacy of suvorexant, or the reason why suvorexant treatment was initiated. Although the baseline dose of BzRA in diazepam equivalents did not differ significantly between the switching and add-on groups, this does not necessarily means that the severity of the patients' conditions was similar in both groups. In terms of the assessment of efficacy, clinical indices (e.g., sTST, clinician global impression of improvement, and polysomnography) could not be employed because of the retrospective nature of this research. Moreover, it is possible that our results might have differed according to the patients' disease status at the time of the prescription of suvorexant; i.e., whether suvorexant was prescribed to reduce the dose of BzRA in a patient with stable disease or to replace a hypnotic. Although the sample size required for the statistical analysis was satisfied, it is necessary to further increase the number of cases to make it possible to adjust for more covariates as described above.

This study provides preliminary evidence regarding the use of suvorexant in insomnia patients taking BzRA. In conclusion, our results show that the add-on of suvorexant increases the risk of oversedation compared with switching in patients taking BzRA. Therefore, when adding suvorexant to BzRA, it is necessary to reduce the dose of BzRA to prevent oversedation although caution should be taken with regard to rebound insomnia. In addition, we expect that suvorexant is a useful adjuvant drug for aiding the withdrawal of benzodiazepines.

\section{REFERENCES}

1. Liu X, Uchiyama M, Kim K, Okawa M, Shibui K, Kudo Y, et al. Sleep loss and daytime sleepiness in the general adult population of Japan. Psychiatry Res 2000;93:1-11.

2. Morin CM, Bélanger L, LeBlanc M, Ivers $\mathrm{H}$, Savard J, Espie CA, et al. The natural history of insomnia: a population-based 3-year longitudinal study. Arch Intern Med 2009;169:447453.

3. Smith E, Narang P, Enja M, Lippmann S. Pharmacotherapy for insomnia in primary care. Prim Care Companion CNS Disord 2016;18:10.4088/PCC.16br01930.

4. Wilt TJ, MacDonald R, Brasure M, Olson CM, Carlyle M, Fuchs E, et al. Pharmacologic treatment of insomnia disorder: An evidence report for a clinical practice guideline by the American College of Physicians. Ann Intern Med 2016;165: 103-112.

5. Siriwardena AN, Qureshi Z, Gibson S, Collier S, Latham M. $G P S$ ' attitudes to benzodiazepine and 'Z-drug' prescribing: a barrier to implementation of evidence and guidance on hypnotics. Br J Gen Pract 2006;56:964-967.

6. Kim HY, Lee HW, Jung SH, Kang MH, Bae JN, Lee JS, et al. Prescription patterns for patients with schizophrenia in Korea: a focus on antipsychotic polypharmacy. Clin Psychopharmacol Neurosci 2014;12:128-136.

7. Wick JY. The history of benzodiazepines. Consult Pharm 2013;28:538-548.

8. Pétursson $\mathrm{H}$. The benzodiazepine withdrawal syndrome. Addiction 1994;89:1455-1459. 
9. Sorock GS, Shimkin EE. Benzodiazepine sedatives and the risk of falling in a community-dwelling elderly cohort. Arch Intern Med 1988;148:2441-2444.

10. Tiihonen J, Suokas JT, Suvisaari JM, Haukka J, Korhonen P. Polypharmacy with antipsychotics, antidepressants, or benzodiazepines and mortality in schizophrenia. Arch Gen Psychiatry 2012;69:476-483.

11. Bertisch SM, Herzig SJ, Winkelman JW, Buettner C. National use of prescription medications for insomnia: NHANES 1999-2010. Sleep 2014;37:343-349.

12. Sakurai T. The neural circuit of orexin (hypocretin): maintaining sleep and wakefulness. Nat Rev Neurosci 2007;8:171181.

13. Norman JL, Anderson SL. Novel class of medications, orexin receptor antagonists, in the treatment of insomnia - critical appraisal of suvorexant. Nat Sci Sleep 2016;8:239-247.

14. Stahl SM. Mechanism of action of suvorexant. CNS Spectr 2016;21:215-218.

15. Michelson D, Snyder E, Paradis E, Chengan-Liu M, Snavely DB, Hutzelmann J, et al. Safety and efficacy of suvorexant during 1-year treatment of insomnia with subsequent abrupt treatment discontinuation: a phase 3 randomised, double-blind, placebo-controlled trial. Lancet Neurol 2014;13: 461-471.

16. Inada T, Inagaki A. Psychotropic dose equivalence in Japan. Psychiatry Clin Neurosci 2015;69:440-447.

17. Kanda Y. Investigation of the freely available easy-to-use soft- ware 'EZR' for medical statistics. Bone Marrow Transplant 2013;48:452-458.

18. Kishi T, Matsunaga S, Iwata N. Suvorexant for primary insomnia: a systematic review and meta-analysis of randomized placebo-controlled trials. PLoS One 2015;10:e0136910.

19. Tyrer P. Risks of dependence on benzodiazepine drugs: the importance of patient selection. BMJ 1989;298:102, 104-105.

20. Herring WJ, Connor KM, Ivgy-May N, Snyder E, Liu K, Snavely $\mathrm{DB}$, et al. Suvorexant in patients with insomnia: Results from two 3-month randomized controlled clinical trials. Biol Psychiatry 2016;79:136-148.

21. Ashton $\mathrm{H}$. The treatment of benzodiazepine dependence. Addiction 1994;89:1535-1541.

22. Voshaar RC, Couvée JE, van Balkom AJ, Mulder PG, Zitman FG. Strategies for discontinuing long-term benzodiazepine use: meta-analysis. Br J Psychiatry 2006; 189:213-220.

23. Rickels K, Schweizer E, Garcia España F, Case G, DeMartinis $\mathrm{N}$, Greenblatt D. Trazodone and valproate in patients discontinuing long-term benzodiazepine therapy: effects on withdrawal symptoms and taper outcome. Psychopharmacology (Berl) 1999;141:1-5.

24. Baandrup L, Lindschou J, Winkel P, Gluud C, Glenthoj BY. Prolonged-release melatonin versus placebo for benzodiazepine discontinuation in patients with schizophrenia or bipolar disorder: A randomised, placebo-controlled, blinded trial. World J Biol Psychiatry 2016;17:514-524. 